\title{
Verhandlungen der Spitaltarife - kantonale Festsetzungen verzerren den Wettbewerb
}

\author{
Der Gesetzgeber lässt im stark regulierten Gesundheitswesen bewusst Freiräume für \\ Marktmechanismen zu. Ein Beispiel sind die Tarifverhandlungen zwischen Kranken- \\ versicherern und Spitälern. Doch inwiefern spielen in diesem wenig transparenten \\ Bereich die geforderten Marktmechanismen tatsächlich? Untersuchungen geben \\ Hinweise, dass kantonale Festsetzungen der Tarife die Entfaltung des Wettbewerbs \\ hemmen und die Verhandlungsergebnisse stark beeinflussen.
}

\section{Jonas Williseggera, Hannes Blatter ${ }^{b}$}

a lic. rer. soc., Senior Researcher, Institut für Betriebs- und Regionalökonomie (IBR), Hochschule Luzern Wirtschaft

b lic. ès sc. pol., Dozent und Projektleiter, Institut für Betriebs- und Regionalökonomie (IBR) Hochschule Luzern Wirtschaft
Korrespondenz: Jonas Willisegger Hochschule Luzern - Wirtschaft Institut für Betriebs- und Regionalökonomie

Frankenstrasse 9

CH-6002 Luzern

Tel. 0412289981
Der gesetzgeberische Wille zum Wettbewerb kommt in gesundheitspolitischen Reformvorhaben immer wieder zum Ausdruck. Aktuell etwa bei der Revision des Heilmittelgesetzes, wonach künftig Apotheken gewisse verschreibungspflichtige Medikamente in eigener Kompetenz verkaufen können und bestimmte Rabatte im Pharmabereich vom grundsätzlichen Rabattverbot ausgenommen werden. Auch im Spitalwesen zielt der Gesetzgeber mit der neuen Spitalfinanzierung und der Einführung von leistungsbezogenen Fallpauschalen auf - im Idealfall - effiziente und transparente Wettbewerbsmechanismen. Diese Haltung wurde unlängst auch vom Bundesverwaltungsgericht bestätigt (Urteil C-1698/2013 vom 7. April 2014), das festhielt, dass Effizienzgewinne von Spitälern nicht KVG-widrig seien.

Die neue Spitalfinanzierung hat insbesondere auch Auswirkungen auf die Tarifverhandlungen zwischen Versicherern und Spitälern. Hier kommt das Mantra des Wettbewerbs besonders stark zum Ausdruck. Doch welche Verhandlungsstrategien werden von welchen Akteuren verfolgt und wie können sich die beabsichtigten Marktdynamiken in den Tarifverhandlungen tatsächlich entfalten?

Ein Forschungsteam am Institut für Betriebs- und Regionalökonomie der Hochschule Luzern - Wirtschaft stellt sich diese Fragen im Rahmen eines laufenden Projektes. Nun liegen die ersten Ergebnisse aus Expertengesprächen und Datenauswertungen zu den Verhandlungsergebnissen der Einkaufsgemeinschaften HSK (Helsana, Sanitas, KPT) und Tarifsuisse vor.

\section{Neue Rahmenbedingungen dank neuer Spitalfinanzierung}

Die Transparenz und Vergleichbarkeit von Daten, die von den Spitälern als Verhandlungsgrundlage an die Krankenversicherer geliefert werden, haben seit dem Systemwechsel zur neuen Spitalfinanzierung spürbar zugenommen. Früher herrschte eine intransparente Subventionierungspraxis durch verschiedene Finanzierungsgefässe, die nicht miteinander verglichen
Négociation des tarifs hospitaliers La fixation des prix par les cantons crée une distorsion

\section{de la concurrence}

Par le nouveau financement hospitalier, le législateur entend faire jouer la concurrence de manière efficace et transparente; ce qui impacte les négociations tarifaires entre assureurs et hôpitaux. Une équipe de chercheurs de l'Institut d'économie d'entreprise et d'économie régionale de la Haute école de gestion de Lucerne a cherché à savoir quelles étaient les stratégies de négociation des principaux acteurs de la santé et à déterminer si la concurrence prévue exercera réellement une influence sur les négociations tarifaires. Les premiers résultats de cette étude sont désormais disponibles.

Le nouveau financement hospitalier pose d'importants défis aux cantons qui doivent composer avec plusieurs casquettes, notamment cofinancer les prestations hospitalières, être propriétaires d'hôpitaux et aussi approuver tous les tarifs hospitaliers. Souvent en effet, ils doivent approuver différents tarifs pour les mêmes prestations hospitalières. Tarifsuisse et HSK, les deux coopératives d'achat qui négocient avec les hôpitaux aux côtés des assureurs, appliquent des stratégies de négociations différentes qui donnent des résultats eux aussi différents. Cet effet de la concurrence entre les partenaires tarifaires est cependant déséquilibré par les interventions cantonales au moment de fixer les prix. 
werden konnten. Heute ist der Kostenteiler zwischen den Krankenkassen und den Kantonen einheitlich definiert.

Dies stellt die Kantone vor gewisse Herausforderungen. Sie haben sich - nach Ablauf der Übergangsfristen - zu 55 Prozent an den Spitalkosten zu beteiligen, sind aber bei den Verhandlungen nicht als Tarifpartner vorgesehen. Die Aufgabe der Kantone ist es jedoch, die für jedes Spital verhandelten Baserates (Basispreise, die mit dem fallspezifischen Kostengewicht der Tarifstruktur SwissDRG multipliziert werden) zu genehmigen oder bei Nicht- das Aufbrechen des Nachfragemonopols bei den Krankenkassen mit sich. Im Jahr 2011 gründete der Branchenverband Santésuisse seine Tochtergesellschaft Tarifsuisse, um ihr die Aufgabe der Tarifverhandlungen im KVG-Bereich zu übertragen. Fast gleichzeitig schlossen sich Helsana, Sanitas und KPT $\mathrm{zu}$ einer neuen, unabhängigen Einkaufsgemeinschaft (HSK) zusammen. Zudem wurde im April 2013 der neue Branchenverband Curafutura gegründet. Dieser vertritt nun die Helsana, CSS, Sanitas und KPT und versteht sich als Verfechter des Wettbewerbs auf Verbandsebene.

\section{Die Tarifpartner nehmen den gesetzgeberischen Willen zu mehr Wettbewerb bisher nur teilweise auf.}

einigung festzusetzen. Erschwerend kommt hinzu, dass viele Kantone Spitaleigentümer sind. Aus Gründen der Strukturerhaltung könnten somit kantonale Gesundheitsdirektorinnen oder -direktoren ein Interesse haben, die Baserates für Spitalleistungen hoch zu halten, um ineffiziente Spitäler indirekt zu subventionieren. Gleichzeitig sind kantonale Finanzdirektoren dem Spardruck ausgesetzt, generell die Gesundheitskosten des Kantons und damit die Baserates tief zu halten.

\section{Aufgebrochenes Nachfragemonopol bei den Krankenkassen}

Dynamik bei den Tarifverhandlungen im Spitalbereich brachte im Grundversicherungsbereich auch

\section{Abbildung 1}

Übersicht über die Verhandlungssettings der ambulanten (TARMED/Taxpunktwerte) und stationären (SwissDRG/Baserates) Tarife.

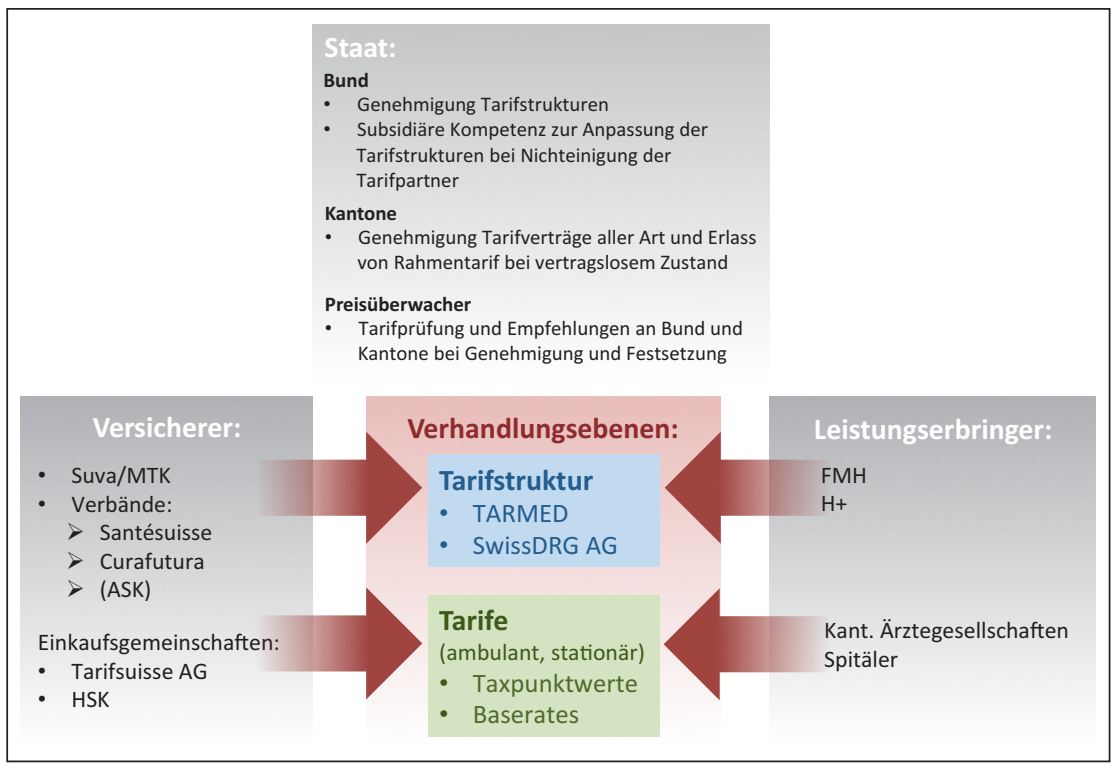

Die aktuellen Rahmenbedingungen und Settings der Verhandlungsebenen, involvierten Tarifpartner und rahmengebenden, subsidiär eingreifenden und genehmigenden öffentlichen Hand lassen sich vereinfacht wie in Abbildung 1 darstellen.

Die Aufsplitterung auf der Einkäuferseite hatte zur Folge, dass die Spitäler in einem Kanton verschiedene Baserates mit Tarifsuisse und HSK (und allfälligen weiteren Verhandlungspartnern) aushandeln können. Manche Kantone hatten zunächst ihre Mühe damit, unterschiedliche Tarife für gleiche Behandlungen zu genehmigen. Die Einkaufsgemeinschaften versuchten sie deshalb zu überzeugen, dass ein Tarifvertrag ein Gesamtpaket mit verschiedenen Komponenten darstellt, die die Tarifunterschiede rechtfertigen können. So lässt sich ein Einjahresvertrag von Tarifsuisse nicht direkt vergleichen mit einem mehrjährigen Gesamtpaket der HSK, das jährlich gestaffelte Tarife und zahlreiche Zusatzvereinbarungen enthält. Marktwirtschaftliche Mechanismen ergeben nicht zwingend Transparenz und Vergleichbarkeit, sondern können auch zu komplexeren Settings bei einer grossen Vielfalt von Angeboten führen.

\section{Unterschiedliche Verhandlungsstrategien}

In einem kompetitiven Umfeld werden Verhandlungsstrategien der involvierten Akteure typischerweise nicht transparent offengelegt. Im vorliegenden Fall können sie aber anhand der Häufigkeit festgesetzter Tarife annähernd identifiziert werden. Kommt es nämlich zwischen einer Einkaufsgemeinschaft und einem Spital aufgrund von kompromisslosen Verhandlungsstrategien zu keiner Einigung, dann setzt der Kanton die Tarife fest. Ein systematischer Vergleich der Verhandlungsergebnisse für sämtliche Schweizer Spitäler seit Einführung der neuen Spitalfinanzierung zeigt, dass Tarifsuisse-Tarife in den Jahren 2012 und 2013 zu rund 40 Prozent festgesetzt wurden, während sich die Tariffestsetzungen bei HSK im tiefen einstel- 


\section{Abbildung 2}

Vergleich der Baserate-Verhandlungsergebnisse von HSK und Tarifsuisse unter Berücksichtigung der Tariffestsetzungen (Grundlage: rund 150 erfasste SwissDRG-Baserates der Schweizer Spitäler gemäss Daten der HSK und Tarifsuisse über die Jahre 2012-2014; Stand: März/April 2014).

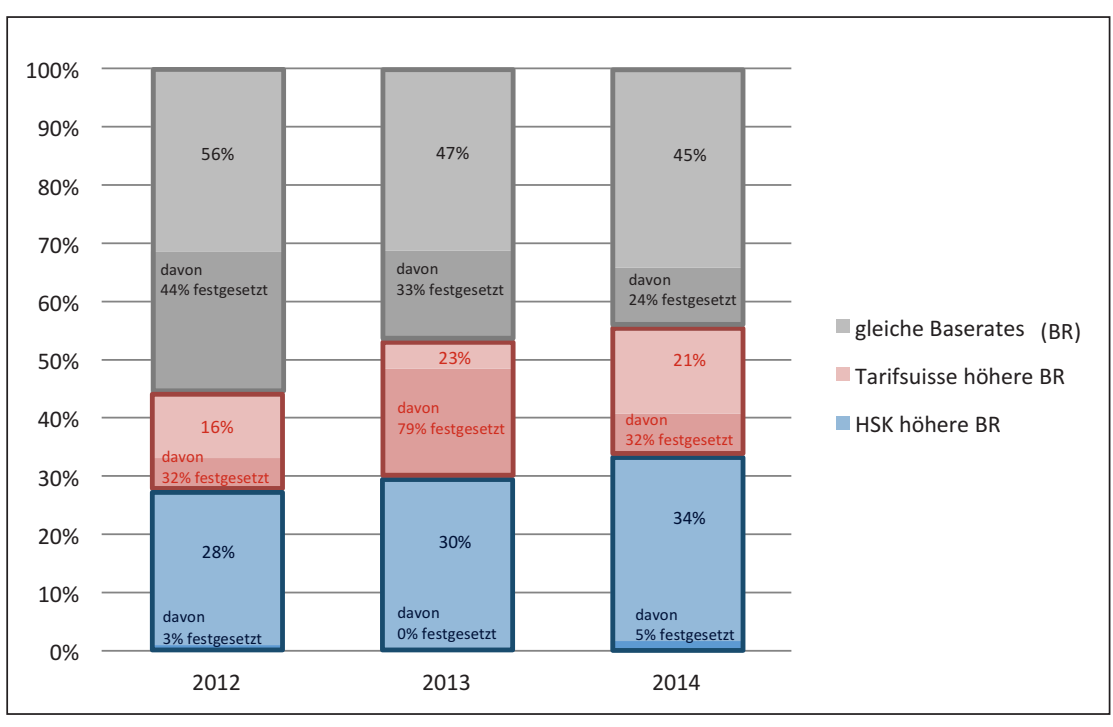

ligen Prozentbereich bewegten. Im Jahr 2014 scheinen die Festsetzungen bei der HSK etwas zu- und bei Tarifsuisse etwas abzunehmen.

Insgesamt weisen die Daten also auf unterschiedliche Verhandlungsstrategien der beiden Einkaufsgemeinschaften hin. Tarifsuisse scheint sich mit den Spitälern seltener einig zu werden als HSK. Dies bestätigen auch Interviewaussagen der Tarifpartner. Sie deuten darauf hin, dass die Spitäler den ersten Verhandlungsschritt oftmals mit HSK machen, bevor sie in die erwartungsgemäss schwierigeren Verhandlungen mit Tarifsuisse treten. Die unterschiedlichen Verhandlungsstrategien der Einkaufsgemeinschaften scheinen also auf dem Markt wahrgenommen zu werden.

\section{Tariffestsetzungen reduzieren Wettbewerbs- effekte und verzerren die Preisbildung}

Schliesslich stellt sich die Frage, ob die unterschiedlichen Verhandlungsstrategien auch zu den erwar-

\section{Interaktiver Artikel}

Wollen Sie diesen Artikel kommentieren? Nutzen Sie dafür die Kommentarfunktion in der OnlineVersion oder sehen Sie nach, was Ihre Kolleginnen und Kollegen bereits geschrieben haben: www.saez.ch/aktuelle-ausgabe/interaktive-beitraege/ teten Ergebnissen führen. Wohl nur bedingt, denn die Untersuchungen zeigen, dass die Tarifpartner den gesetzgeberischen Willen zu mehr Wettbewerb bisher nur teilweise aufnehmen. In rund der Hälfte aller Schweizer Spitäler sind die Baserates von Tarifsuisse und HSK gleich hoch, obwohl im System beträchtlicher Verhandlungsspielraum identifiziert werden kann. Höhere HSK-Baserates sind in über 95 Prozent der Fälle Ergebnis freier Verhandlungen, während höhere Tarifsuisse-Tarife sich in 30 bis 80 Prozent der Fälle (je nach Jahr) auf kantonale Festsetzungen zurückführen lassen (Abb. 2). Wobei die Einschränkung gilt, dass die beiden Tarife nur bedingt miteinander verglichen werden können. Die kantonal festgesetzten Tarife sind provisorisch, denn Tarifsuisse hat in den meisten Fällen Beschwerde beim Bundesverwaltungsgericht eingelegt. Dessen Entscheide sind noch hängig.

Werden die Differenzen zwischen den Tarifen unter Vorbehalt der oben formulierten Einschränkung - unter die Lupe genommen, lässt sich erkennen, dass in denjenigen Spitälern, wo die TarifsuisseBaserates höher sind, die Differenzen zwischen Tarifsuisse und HSK bedeutend grösser ausfallen als dort, wo die HSK-Baserates höher sind. Die grössten Differenzen ergeben sich in den Kantonen mit Universitätsspitälern. Deren Tarife sind oftmals auf Festsetzungen zurückzuführen.

So kann die These aufgestellt werden, dass die Wettbewerbsentfaltung durch staatliche, subsidiäre Eingriffe in die Preisbildung mittels kantonalen Festsetzungen gehemmt und das Verhandlungsergebnis stark verzerrt wird. Denn Festsetzungen führen häufig zu Beschwerden und zu Bundesverwaltungsgerichtsentscheiden, die ihrerseits starken Einfluss auf die Rahmenbedingungen der Verhandlungen und damit auf die Preise haben. Ob das heutige Verfahren insbesondere der Tariffestsetzungen mit den Zielen der neuen Spitalfinanzierung (Transparenz, Vergleichbarkeit, Effizienz) noch in Einklang steht oder grundsätzlich überdacht werden soll, gilt es vertieft zu untersuchen.

Dank der finanziellen Unterstützung durch die Stiftung Konkordia konnte im März 2012 an der Hochschule Luzern - Wirtschaft ein mehrjähriges Forschungsprogramm «Öffentliches Gesundheitsmanagement» gestartet werden (www.hslu.ch/w-ibr-oeffentlichesgesundheitsmanagement.htm). 